\title{
OPEN Porcine rotavirus $B$ as primary causative agent of diarrhea outbreaks in newborn piglets
}

\author{
Flavia Megumi Miyabe ${ }^{1,2}$, Alais Maria Dall Agnol ${ }^{1,2}$, Raquel Arruda Leme ${ }^{1,2}$, \\ Thalita Evani Silva Oliveira ${ }^{3}$, Selwyn Arlington Headley ${ }^{3}$, Thiago Fernandes ${ }^{4}$, \\ Admilton Gonçalves de Oliveira ${ }^{4}$, Alice Fernandes Alfieri ${ }^{1,2}$ \& Amauri Alcindo Alfieri ${ }^{1,2 \bowtie}$
}

Rotavirus (RV) is considered a major cause of acute viral gastroenteritis in young animals. RV is classified into nine species, five of which have been identified in pigs. Most studies worldwide have highlighted diarrhoea outbreaks caused by RVA, which is considered the most important RV species. In the present study, we described the detection and characterization of porcine RVB as a primary causative agent of diarrhoea outbreaks in pig herds in Brazil. The study showed a high frequency $(64 / 90 ; 71.1 \%)$ of RVB diagnosis in newborn piglets associated with marked histopathological lesions in the small intestines. Phylogenetic analysis of the VP7 gene of wild-type RVB strains revealed a high diversity of $\mathrm{G}$ genotypes circulating in one geographic region of Brazil. Our findings suggest that RVB may be considered an important primary enteric pathogen in piglets and should be included in the routine differential diagnosis of enteric diseases in piglets.

Rotaviruses (RV) are members of the Reoviridae family and a major cause of acute viral gastroenteritis in young animals, including piglets ${ }^{1}$. RV is a non-enveloped virus and its genome is composed of 11 segments of doublestranded $\mathrm{RNA}^{2}$. RV is classified into nine species (A-I), on the basis of the antigenic and genetic characteristics of viral protein $6(\mathrm{VP} 6)^{3,4}$. A new tentative species J has been recently identified in bats from Serbia ${ }^{5}$. Currently, five of the nine species of RV have been identified in pigs (RVA, RVB, RVC, RVE, and RVH) ${ }^{6}$.

RVA was the first RV species to be identified and is considered the most important RV species because of its high prevalence and pathogenicity in both humans and animals ${ }^{1,7}$. In 2008, a nucleotide sequence-based classification system for the complete genome of RVA strains was developed; this system assigned specific genotypes to each of the $11 \mathrm{RV}$ genome segments according to nucleotide (nt) and amino acid (aa) percent cutoff values ${ }^{8,9}$. The Rotavirus Classification Working Group (RCWG) maintains this classification and uses the abbreviations Gx-P[x]-Ix-Rx-Cx-Mx-Ax-Nx-Tx-Ex-Hx for the RV genes VP7-VP4-VP6-VP1-VP2-VP3-NSP1-NSP2-NSP3NSP4-NSP5/6, respectively ${ }^{8,10}$.

Unlike RVA strains, which have been well characterized worldwide, RVB strains remain poorly characterized. In 2012, Marthaler et al. ${ }^{11}$ proposed a classification for G genotypes of RVB strains from the United States (USA), establishing $20 \mathrm{G}$ genotypes; however, the complete genome of porcine RVB was described only recently ${ }^{12}$. Shepherd et al. ${ }^{12}$ established a provisional genome-based classification for RVB, suggesting and updating nt cutoff values for all RVB gene segments. According to this newly proposed classification, 26G, 5P, 13I, 5R, 5C, 5M, 8A, $10 \mathrm{~N}, 6 \mathrm{~T}$, 4E, and 7H genotypes (VP7, VP4, VP6, VP1-VP3, NSP1-NSP5 genes, respectively) of RVB have been described, using nt cutoff values of $80 \%, 80 \%, 81 \%, 78 \%, 79 \%, 77 \%, 76 \%, 83 \%, 78 \%, 76 \%$, and $79 \%$, respectively.

Studies conducted in the USA and Brazil have shown high frequencies of RVB in piglets with diarrhoea, mostly in association with other RV species, such as RVA and $\mathrm{RVC}^{6,7,11}$. Thus, the role of RVB as a primary pathogen remains unclear, and until recently, this species was not considered a major causative agent of diarrhoea in piglets.

In the present study, we describe the detection of porcine RVB acting as a potential primary causative agent of neonatal diarrhoea outbreaks in several pig farms from the Central-West region of Brazil and the characterization of G genotypes of these Brazilian RVB field strains.

\footnotetext{
${ }^{1}$ Laboratory of Animal Virology, Department of Preventive Veterinary Medicine, Universidade Estadual de Londrina, PO Box 10011, Londrina, Paraná 86057-970, Brazil. ${ }^{2}$ Multi-User Animal Health Laboratory-Molecular Biology Unit, Department of Preventive Veterinary Medicine, Universidade Estadual de Londrina, Londrina, Paraná, Brazil. ${ }^{3}$ Laboratory of Animal Pathology, Department of Veterinary Preventive Medicine, Universidade Estadual de Londrina, Londrina, Paraná, Brazil. ${ }^{4}$ Laboratory of Electron Microscopy, Department of Microbiology, Universidade Estadual de Londrina, Londrina, Paraná, Brazil. ${ }^{\circledR}$ email: alfieri@uel.br
} 


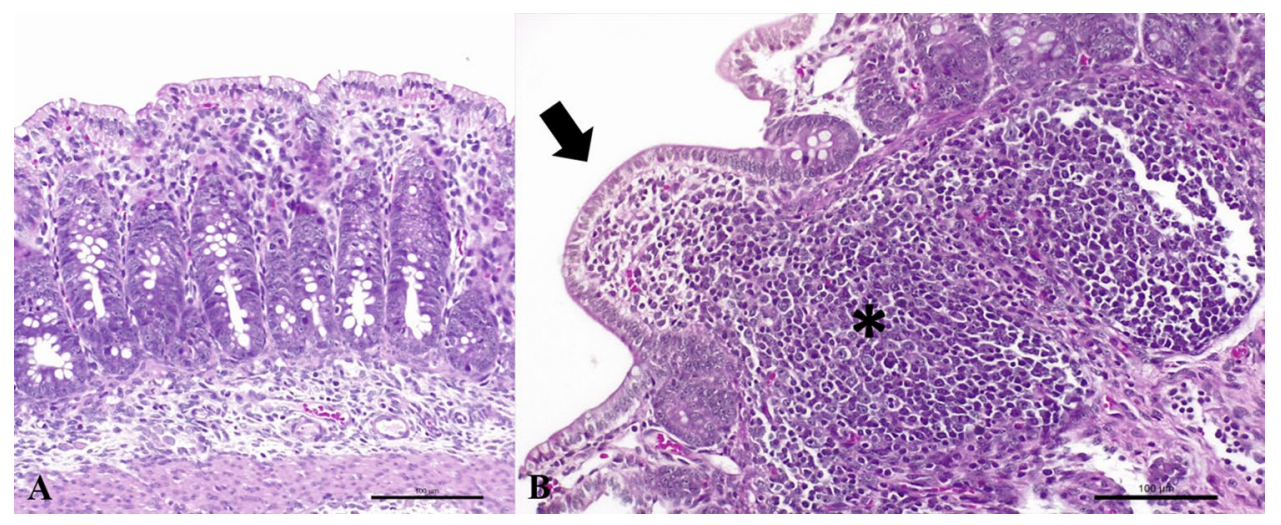

Figure 1. Histopathological findings in jejunum observed in newborn piglets naturally infected by Rotavirus B. (A) Observed marked atrophic enteritis with villous atrophy and blunting. (B) Severe atrophic enteritis with villous fusion (arrow) and hyperplasia of Peyer patches (asterisk). Hematoxylin \& Eosin stain. Bar, $(\mathbf{A}, \mathbf{B})$ $100 \mu \mathrm{m}$. Magnification, $\times 40$.

\begin{abstract}
Results
PAGE and conventional RT-PCR. Forty-two of the 90 (46.7\%) biological samples evaluated in this study displayed typical electropherotype of RVB species on polyacrylamide gel electrophoresis assay (PAGE), while 14 samples had inconclusive results. In the conventional RT-PCR assay, 64 of the $90(71.1 \%)$ samples were positive for RVB; these corresponded to $46.2 \%$ (6/13) of the intestinal tissues and $75.3 \%(58 / 77)$ of the diarrheic faeces samples evaluated. Coinfections of RVB with RVA and RVH were identified, by conventional RT-PCR, in $2.22 \%$ $(2 / 90)$ and $4.44 \%$ (4/90) of samples, respectively. All the following enteric viruses were negative in all samples by conventional RT-PCR: RVC, swine delta coronavirus (SDCoV), porcine epidemic diarrhoea virus (PEDV), transmissible gastroenteritis virus (TGEV), Seneca Valley virus (SVV), and porcine enterovirus (PEV).
\end{abstract}

Histopathology. The histopathological findings from the jejunum revealed atrophic enteritis with marked villous atrophy and blunting, as well as reduced crypt depth, submucous lymphangiectasis, and hyperplasia of Peyer patches (Fig. 1).

Transmission electron microscopy. The electron microscopy (EM) examination of a faecal sample revealed the presence of wheel-shaped viruses of approximately $70 \mathrm{~nm}$, similar to RV-like particles (Supplementary Fig. S1).

Virus isolation. Attempts at virus isolation from cell culture were performed using the same faecal sample that was analysed by EM; however, after nine blind passages in monolayer of MA-104 (African green monkey kidney) cells, no cytopathic effect (CPE) was visualized. Moreover, aliquots of all passages tested negative for RVB and other RV species (RVA, RVC and RVH), as well as SDCoV, PEDV, TGEV, SVV, and PEV in the RT-PCR assay.

Real-time reverse transcription PCR assay (qRT-PCR). The qRT-PCR assay developed in this study for quantification of RVB RNA in piglet faecal samples had a limit of detection (LOD) of 13.4 genomic copies/ $\mu \mathrm{L}$. The viral loads in diarrheic faecal samples varied from $7.6 \times 10^{2}$ to $8.5 \times 10^{8}$ genomic copies/g of faeces. The mean viral loads of the RVB-positive samples are described in terms of pig herd and sampling in Table 1. In pig herds A and B, the RVB viral load shed in the faeces of diarrheic piglets seems to have a cyclic pattern, with high and low viral loads observed in the four samplings during the outbreak.

Sequencing and phylogenetic analysis. Comparative sequence analysis of the VP7 gene of 16 RVB field strains found in this study and the 26 known $G$ genotypes of RVB $^{12}$ revealed that at least four different $G$ genotypes (G12, G14, G16, and G20) were circulating in these outbreaks.

The GO-949, GO-951, GO-1099, and GO-1113 strains displayed higher (77.1 to 80.4\%) nt identity with other G12 strains; the GO-936 strain displayed 80 to $84.4 \%$ nt identity with the G14 strains; and the GO-992 strain displayed 81.3 to $85.4 \%$ nt identity with the G20 strains. Last, the GO-908, GO-923, GO-968, GO-970, GO-983, GO-1045, GO-1053, GO-1119, GO-1123, and GO-1127 strains showed higher (81.5 to 83.9\%) nt identity with the G16 strains, which was the most prevalent G genotype found in this study (Supplementary Table S2 shows the identity matrix of RVB strains described in this study and representative strains of the 26 previously identified RVB G genotypes $\left.{ }^{12}\right)$.

In the phylogenetic tree (Fig. 2), the G16 RVB Brazilian field strains clustered in a different branch in comparison with other G16 strains; however, using the nt cutoff value of $80 \%$, the sequence identity matrix revealed that the strains belong to the same G genotype. Comparison among the Brazilian G12 strains showed that the 


\begin{tabular}{|c|c|c|c|c|c|}
\hline Pig herd & Sampling & Month/year of sampling & RVB positive/total samples & $\begin{array}{l}\text { Mean viral load (genomic copies/g } \\
\text { of faeces) }\end{array}$ & G genotype \\
\hline \multirow{4}{*}{ A } & 1 & Aug/2017 & $3 / 4$ & $1.7 \times 10^{8}$ & G16 \\
\hline & 2 & Oct/2017 & $1 / 1$ & $1.3 \times 10^{5}$ & - \\
\hline & 3 & Feb/2018 & $5 / 5$ & $1.7 \times 10^{8}$ & G16 \\
\hline & 4 & Aug/2018 & $0 / 5$ & Not tested & - \\
\hline \multirow{4}{*}{ B } & 1 & Aug/2017 & $5 / 6$ & $2.0 \times 10^{8}$ & G16 \\
\hline & 2 & Oct/2017 & $1 / 1$ & $2.5 \times 10^{5}$ & - \\
\hline & 3 & Feb/2018 & $8 / 9$ & $1.9 \times 10^{7}$ & G16 \\
\hline & 4 & Aug/2018 & $3 / 5$ & $2.3 \times 10^{3}$ & - \\
\hline \multirow{5}{*}{$\mathrm{C}$} & 1 & Aug/2017 & $5 / 5$ & $8.9 \times 10^{7}$ & G16 \\
\hline & 2 & Sep/2017 & $5 / 5$ & $6.6 \times 10^{7}$ & G16 \\
\hline & 3 & Oct/2017 & $1 / 1$ & $2.2 \times 10^{7}$ & G16 \\
\hline & 4 & Jun/2018 & $3 / 4$ & $2.6 \times 10^{3}$ & G12 \\
\hline & 5 & Aug/2018 & $0 / 2$ & Not tested & - \\
\hline \multirow{5}{*}{$\mathrm{D}$} & 1 & Aug/2017 & $1 / 5$ & $3.6 \times 10^{7}$ & G14 \\
\hline & 2 & Sep/2017 & $2 / 2$ & $7.6 \times 10^{6}$ & - \\
\hline & 3 & Oct/2017 & $1 / 3$ & $4.3 \times 10^{5}$ & - \\
\hline & 4 & Jun/2018 & $3 / 3$ & $5.0 \times 10^{7}$ & G16 \\
\hline & 5 & Aug/2018 & $0 / 5$ & Not tested & - \\
\hline $\mathrm{E}$ & 1 & Sep/2017 & $10 / 12$ & $1.1 \times 10^{7}$ & G12 \\
\hline F & 1 & Oct/2017 & $1 / 1$ & $3.5 \times 10^{7}$ & G20 \\
\hline G & 1 & Jun/2018 & $1 / 1$ & $9.1 \times 10^{7}$ & G16 \\
\hline $\mathrm{H}$ & 1 & Jun/2018 & $5 / 5$ & $2.1 \times 10^{3}$ & G12 \\
\hline Total & & & $64 / 90$ & & \\
\hline
\end{tabular}

Table 1. Distribution of total biological samples positives for RVB and respective viral load and G genotypes identified by pig herds and sampling.

strains share $85.4-99.8 \%$ nt identity, while the strains belonging to the G16 genotype shared high (96.6 to $100 \%)$ nt identity.

As the outbreaks occurred over a period of one year, some samples collected from pig herds were from different peaks of diarrhoea episodes, and it was possible to longitudinally analyse these RVB strains. Two different $\mathrm{G}$ genotypes were found circulating in pig herds $\mathrm{C}$ and $\mathrm{D}$ over the sampling period. In pig herd C, the genotype G16 was detected in the first samplings, while in the last sampling, the G12 genotype was identified. Alternatively, in pig herd D, the genotype G14 was identified in the first samplings, while the genotype G16 was detected in the last samplings (Table 1).

\section{Discussion}

Although RVA has been reported as the most prevalent RV species in both humans and animals, porcine RVB was previously described in several regions worldwide, but usually in sporadic cases ${ }^{13-15}$. Studies conducted in Japan, the USA, and Brazil have shown porcine RVB frequencies of $25.9 \%, 46.8 \%$, and 32\%, respectively $y^{6,11,16}$. However, in these previous reports, RVB was detected mostly in association with other RV species, such as RVA, RVC, and RVH. In the present study, we detected porcine RVB in more than $70 \%(64 / 90)$ of the diarrheic faeces/ intestine tissues, with the highest RVB frequency reported thus far. In addition, most (58/90; 64.4\%) of the diarrheic faecal samples were positive only for RVB, and coinfections with other RV species were detected in only $6(6.7 \%)$ faecal samples. Furthermore, other important enteric viruses (RVC, SDCoV, PEDV, TGEV, SVV, and PEV) were not detected in these samples, and diarrhoea outbreaks were unresponsive to treatment with wide-spectrum antibiotics.

The high mortality rate observed in the outbreaks reported in this study suggests the involvement of pathogenic RVB field strains. The marked villous atrophy observed in the small intestines of affected piglets resulted in acute malabsorption and diarrhoea, which may have led to the high mortality of piglets. Infections caused by SVV and TGEV may produce similar lesions in the intestines of affected pigs ${ }^{17-19}$. Nevertheless, in this study, these viral disease pathogens were not identified by RT-PCR. It must be highlighted that RV replicates predominantly in the cytoplasm of villous enterocytes of the jejunum and ileum and induces cell lysis, villous blunting and atrophy, as observed by histopathology. Severe lesions are mostly associated with the RVA and RVC species ${ }^{20,21}$; however, our results suggest that RVB may also induce marked lesions in the small intestine of affected piglets and can be considered an important enteric pathogen in pigs, acting as a primary aetiologic agent of neonatal diarrhoea outbreaks.

Kuga et al. ${ }^{16}$ and Marthaler et al. ${ }^{11}$ observed the highest frequencies of RVB in animals $>55$ days of age or fattening pigs, implying that RVB detection in clinical samples appears to increase with age. On the other hand, Alekseev et al. ${ }^{22}$ detected RVB causing a diarrhoea outbreak in suckling piglets with 3 to 5 days of age in Russia, 


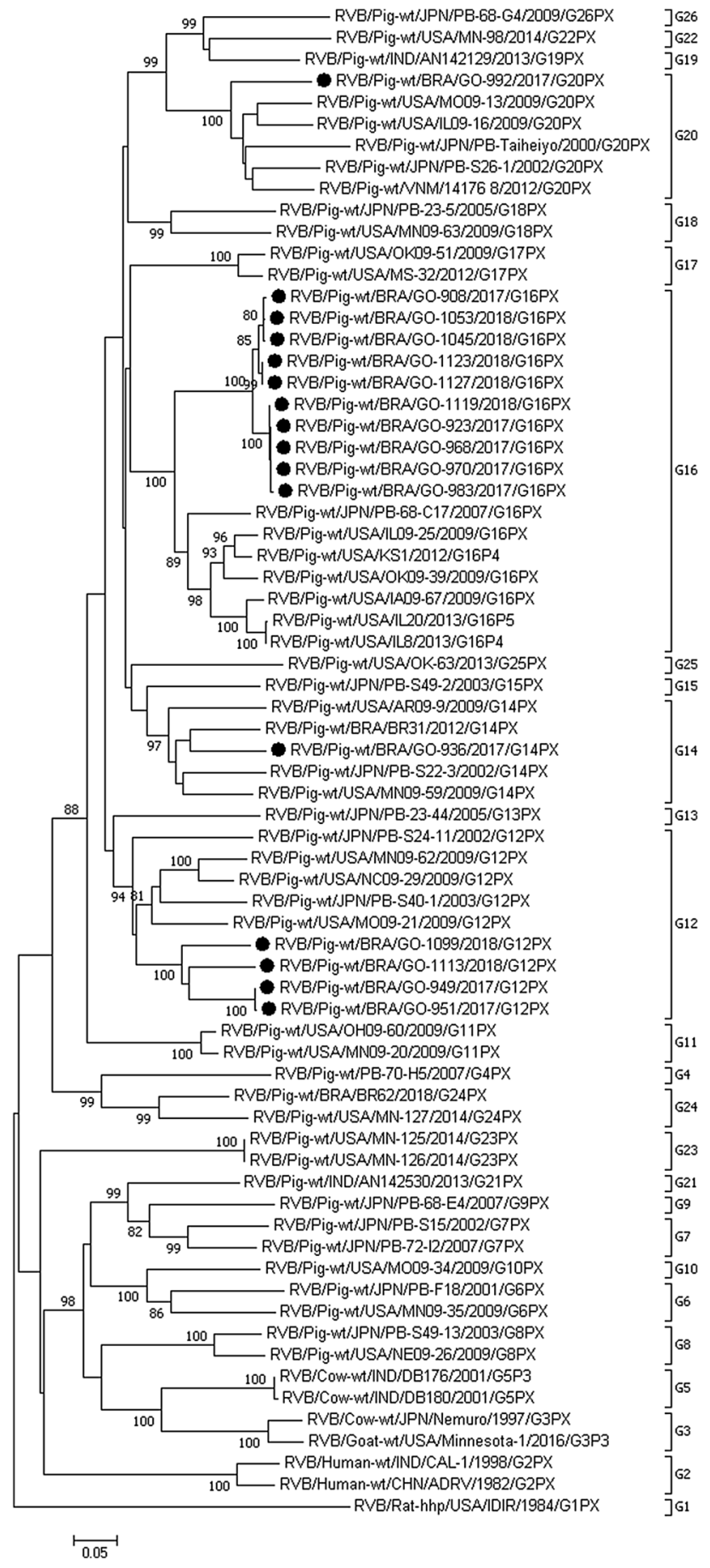

Figure 2. Phylogenetic tree based on nucleotide sequences (700 nt) of the VP7 gene of porcine RVB strains described in this study and representative strains of the 26 previously identified RVB G genotypes ${ }^{12}$. The tree was constructed using the neighbor-joining method based on the Kimura 2-parameter model. The scale bar indicates nt substitutions per site. The bootstrap values (1000 replicates) are shown at the branch nodes (values $<80 \%$ not shown). The Brazilian porcine RVB strains are marked with filled circles.

corroborating our results. The diarrhoea outbreaks described in our study affected only piglets $<20$ days of age, mostly newborn piglets, suggesting that RVB can be highly detected in all age groups.

Some authors have suggested that the immune pressure induced by mass vaccination against RVA may have driven the selection and emergence of other RV species, such as RVB and RVC ${ }^{23,24}$. The high frequency of RVB found in this study contributes to this hypothesis since the sows of all pig herds described in this study were routinely vaccinated against enteric pathogens, including RVA. Molinari et al. ${ }^{6}$ reported similar findings describing 
the detection of multiple RV groups in single and mixed infections in an unusual post-weaning porcine diarrhoea outbreak in a pig herd vaccinated with the RVA OSU strain in Brazil.

It is known that RVA species are shed in large quantities in stools of infected animals during episodes of diarrhoea, with viral loads of approximately $10^{12}$ viral particles per gram of faeces ${ }^{25}$. In this study, the RVB viral load found was up to $8.5 \times 10^{8}$ genomic copies/g of faeces, suggesting that lower quantities of RVB are shed during the acute period of infection compared to RVA. However, few virions are needed to cause disease in susceptible animals ${ }^{4}$ and the transmission of RV infection occurs predominantly by the faecal-oral route, suggesting that environmental contamination contributed to the various outbreaks that occurred during the total outbreak period described in the present study.

The low prevalence of RVB reported worldwide may not be a consequence of a low infection rate but rather due to diagnostic gaps ${ }^{26}$. Some techniques, such as ssPAGE and EM, have low sensitivity, presenting an LOD of approximately $10^{6}$ particles/ $\mathrm{mL}^{27}$. In this study, the prevalence of RVB found by conventional RT-PCR was higher than by ssPAGE. Moreover, the viral loads found in this study varied from $7.6 \times 10^{2}$ to $8.5 \times 10^{8}$ genomic copies/g of faeces, and most of the positive samples on ssPAGE presented viral loads above $10^{6}$ genomic copies/g of faeces (data not shown). Therefore, the use of more sensitive diagnostic techniques is necessary to avoid false-negative results. Molecular techniques, such as RT-PCR and qRT-PCR, usually present high sensitivity and are suitable assays to detect RVB from porcine biological samples ${ }^{6,7,23}$.

Unlike RVA, RVB does not adapt and propagate in cell culture, which has hindered its serological and molecular characterization, as well as the development of vaccines against RVB disease. In 1996, the isolation of a putative RVB strain in cultured cells, designated the SKA-1 strain, was reported ${ }^{28}$. However, some years later, the same research group found that this strain was actually more closely related to $\mathrm{RVH}^{29}$. In this study, our attempts to isolate RVB in cell culture were unsuccessful. Nevertheless, by EM, we identified RV-like particles in the same sample that was submitted for virus isolation in cell culture. Electron microscopy lacks specificity because it cannot differentiate among RV species²; however, the submitted sample tested negative in the RT-PCR for all RV species and other viral agents, except for RVB, suggesting that the virus identified was probably RVB. In addition, the VP7 protein of this RVB strain was amplified, and we could determine its G genotype (G20).

Until recently, information regarding the genome of porcine RVB strains was restricted to only a few proteins $^{11,16,30-33}$. The classification for G genotypes was first proposed by Kuga et al. ${ }^{16}$, who analysed the VP7 gene of Japanese RVB strains. Using nt cutoff values of 67 and 76\%, the authors established five G genotypes, divided into 12 clusters ${ }^{16}$. After that, based on the analysis of RVB strains from the USA, Marthaler et al. ${ }^{11}$ proposed a modification of the nt cutoff value to $80 \%$, establishing twenty $G$ genotypes. Based on this new cutoff value, Lahon et al. ${ }^{14}$ and Molinari et al. ${ }^{34}$ each described new RVB G genotypes, G21 and G22, respectively. More recently, Shepherd et al. ${ }^{12}$ described the whole genome of USA RVB strains, suggesting and updating cutoff values for all RVB gene segments, including those that had not been established yet. According to this most recent classification, $26 \mathrm{G}$ genotypes for RVB were defined. In addition, the previously reported Brazilian G22 strain ${ }^{34}$ was reclassified into the G24 genotype.

Only two VP7 RVB strains have been described in Brazil thus far, BR31 (G14) and BR62 (G24) ${ }^{6,34}$. In this study, we report 16 new Brazilian VP7 RVB wild-type strains. The phylogenetic analysis performed with the complete VP7 gene of the RVB strains identified in this study revealed that four different G genotypes were circulating in the same Brazilian geographic region (Central-West). The G16 genotype was the most prevalent, followed by the G12, G20, and G14 genotypes. Marthaler et al. ${ }^{11}$ also reported G16 as the most prevalent G genotype in RVB field strains in the USA.

Interestingly, in the two pig herds from which we performed longitudinal phylogenetic analysis, two different $G$ genotypes were detected over the sampling period. In pig herd C, the G16 genotype was detected within the first to third samplings, while in the fourth sampling, which occurred 8 months later, the G12 genotype was found. Similarly, in pig herd D, the genotype found in the first sampling was G14, and the G16 genotype was found circulating in the same herd ten months later. These findings suggest a high variation in $G$ genotypes circulating in Brazil.

RVB appears to be more diverse than RVA and RVC when considering the number of genotypes in swine hosts. According to Shepherd et al. ${ }^{12}, 17 \mathrm{G}$ genotypes of RVB have been identified in pigs compared with 12 and 15 G genotypes of RVA and RVC, respectively. Some authors even suggested that swine species might be the original natural host of RVB, strongly contributing to the genetic diversity of this RV species ${ }^{16,35}$.

Since RVB has been identified as an important enteric pathogen of pigs, it is essential to incorporate screening for RVB along with RVA and RVC in the diagnostic routine of swine enteric diseases. More information about the epidemiology of porcine RVB is needed to better understand its pathogenicity and prevent RVB infections, since to date there are no vaccines available for this RV species.

\section{Conclusion}

This study reports RVB as the primary causative agent of neonatal diarrhoea outbreaks in newborn piglets. RVB was associated with marked villous atrophy and blunting in the jejunum of affected piglets, inducing malabsorptive diarrhoea and leading to high mortality rates. We suggest that the reported repeated outbreaks in these pig herds were associated with environmental contamination since we found mild to high genomic copies of RVB in piglet faeces. Additionally, the rates of RVB diagnosis in diarrhoea outbreaks reported in this study were much higher than those described in previous studies, mainly in single infections. Moreover, the variety of $\mathrm{G}$ genotypes identified in these outbreaks suggests that genetic diversity among porcine RVB field strains may be underestimated. Further studies are necessary to investigate the prevalence of RVB in other regions of Brazil and in other countries, as well as to identify the genotypes circulating worldwide. 


\begin{tabular}{|c|c|c|c|c|c|}
\hline Virus & Viral gene & Primer sequence $\left(5^{\prime}-3^{\prime}\right)$ & Primer position & RT-PCR product (bp) & References \\
\hline RVA & $\begin{array}{l}\text { VP4 } \\
\text { VP7 }\end{array}$ & $\begin{array}{l}\text { Fw-TGGCTTCGCCATTTLATAGACA } \\
\text { Rv-ATTTCGGACCATTTATAACC } \\
\text { Fw-GGCTTTAAAAGAGAGAATTTCCGTCTGG } \\
\text { Rv-GGTCACATCATACAATTCTAATCTAAG }\end{array}$ & $\begin{array}{l}12-33 \\
868-887 \\
1-21 \\
1038-1062\end{array}$ & $\begin{array}{l}876 \\
1062\end{array}$ & $\begin{array}{l}39 \\
40\end{array}$ \\
\hline RVB & NSP2 & $\begin{array}{l}\text { Fw-CTATTCAGTGTGTCG TGAGAGG } \\
\text { Rv-CGAAGCGGGCTAGCTTGTCTGC }\end{array}$ & $\begin{array}{ll}1-18 \\
434-451\end{array}$ & 434 & 41 \\
\hline RVB & VP7 & $\begin{array}{l}\text { Fw-GCGTTGCCACTGCTTCTC } \\
\text { Rv-TTTTTATTGGCTTCGGCTACTC }\end{array}$ & $\begin{array}{ll}18-35 \\
796-817\end{array}$ & 800 & 15 \\
\hline RVC & VP6 & $\begin{array}{l}\text { Fw-GGCTTTAAAAATCTCATTCA } \\
\text { Rv-CCTCTAGTTGATTGAACATA }\end{array}$ & $\begin{array}{ll}1-20 \\
251-270\end{array}$ & 270 & 42 \\
\hline RVH & VP6 & $\begin{array}{l}\text { Fw-ACCAGGTGGAGCAACAAACA } \\
\text { Rv-CAGTGCGTGACCAGATCTCA }\end{array}$ & $\begin{array}{l}529-549 \\
1225-1244\end{array}$ & 716 & 43 \\
\hline SDCoV & M & $\begin{array}{l}\text { Fw-ATCCTCCAAGGAGGCTATGC } \\
\text { Rv-GCGAATTCTGGATCGTTGTT }\end{array}$ & $\begin{array}{l}67-87 \\
540-560\end{array}$ & 493 & 44 \\
\hline TGEV & $S$ & $\begin{array}{l}\text { Fw-GTGGTTTTGGTYRTAAATGC } \\
\text { Rv-CACTAACCAACGTGGARCTA }\end{array}$ & $\begin{array}{l}16-35 \\
855-874\end{array}$ & 859 & 45 \\
\hline PEDV & S & $\begin{array}{l}\text { Fw-TTCTGAGTCACGAACAGCCA } \\
\text { Rv-CATATGCAGCCTGCTCTGAA }\end{array}$ & $\begin{array}{l}1466-1485 \\
2097-2116\end{array}$ & 651 & 45 \\
\hline SVV & VP1 & $\begin{array}{l}\text { Fw-ACTGACACCGATTTCTCTG } \\
\text { Rv-CTAAAGTAAGTGAAACAGGC }\end{array}$ & $\begin{array}{l}2730-2749 \\
3026-3046\end{array}$ & 316 & 46 \\
\hline PEV & 5' NTR & $\begin{array}{l}\text { Fw-CAAGCACTTCTGTTTCCCCGG } \\
\text { Rv-GTTAGGATTAGCCGCATTCAGGGG }\end{array}$ & $\begin{array}{l}197-271 \\
486-509\end{array}$ & 313 & 47 \\
\hline
\end{tabular}

Table 2. Gene targets and respective RT-PCR products used to identify enteric viruses in pig samples.

\section{Materials and methods}

Herds and samples. Neonatal diarrhoea outbreaks occurred from July 2017 to August 2018 in piglet production units from eight pig herds located in a single state of the Central-West region of Brazil. The all-in-all-out production system was used for all the pig herds, and good nutritional and health management practices were used, including the addition of vitamin and probiotic supplements and heat placement for newborn piglets. All sows were vaccinated with a commercial vaccine containing the RVA OSU (G5P [7]) strain, Escherichia coli (K88, K99, F41 and 987P) and Clostridium perfringens types C and D, following the manufacturer's recommendations. Nevertheless, the outbreaks were unresponsive to wide-spectrum antibiotic therapy, exhibiting high mortality (10 to 50\%) and morbidity ( 35 to $50 \%$ ) rates. Piglets up to 20 days of age, mostly newborn piglets, from all herds were affected and exhibited dehydration and watery diarrhoea, with the onset of symptoms usually at one day after birth.

For four of the eight pig herds affected by the diarrhoea outbreak, four or five sampling were collected at different times, with 1-8 months between each sampling, enabling us to perform a longitudinal analysis of these herds.

A total of 90 biological samples, including 77 diarrheic faeces and 13 fragments of the small intestines from suckling piglets ( 1 to 20 days of age), were subjected to analysis for the identification of enteric viruses. All intestinal fragments were immersed in $10 \%$ buffered formalin solution and then routinely processed for histopathologic evaluation with haematoxylin and eosin (H\&E) staining.

Nucleic acid extraction, PAGE, and conventional RT-PCR. The nucleic acid was extracted from $10 \%$ faecal/intestine suspensions in phosphate-buffered saline (PBS), pH 7.2, using a combination of phenol/chloroform/isoamyl alcohol (25:24:1) and silica/guanidine isothiocyanate methods, described by Alfieri et al. ${ }^{36}$. The nucleic acid was eluted in $50 \mu \mathrm{L}$ of UltraPure DEPC-treated water (Invitrogen Life Technologies, Carlsbad, CA, USA) and stored at $-80^{\circ} \mathrm{C}$ until further use. Aliquots of ultrapure sterile water were included in all nucleic acid extraction and the following procedures as negative controls.

All the samples were tested by PAGE assay to verify the presence of $\mathrm{RV}^{13}$. The extracted nucleic acid was also subjected to conventional RT-PCR assay to investigate and confirm the presence of RV species A, B, C, and H, as well as other porcine enteric viruses, such as SDCoV, PEDV, TGEV, SVV, and PEV. The gene targets and the RT-PCR products of each virus are described in Table 2 . The amplified products were analysed by $2 \%$ agarose gel electrophoresis in TBE buffer; the gels were stained with $0.5 \mu \mathrm{g} / \mathrm{mL}$ of ethidium bromide and visualized under ultraviolet light.

Transmission electron microscopy. Two faecal samples positive for RVB in the RT-PCR assay were subjected to EM examination. The $10 \%(\mathrm{w} / \mathrm{v})$ faecal suspensions in PBS solution were clarified by centrifugation at $1500 \times g$ for $30 \mathrm{~min}$. Then, a $5 \mu \mathrm{L}$ drop of each sample was applied onto a 300 -mesh formvar-coated cooper grid (Electron Microscopy Sciences, Hatfield, PA, USA) and negatively stained with $3 \%$ phosphotungstic acid for $90 \mathrm{~s}$. Grids were prepared in duplicate and examined at $80 \mathrm{kV}$ with a Tecnai-G2 transmission electron microscope (FEI Company, Eindhoven, The Netherlands). 
Virus isolation. Attempts at virus isolation from two RVB-positive faecal samples in the RT-PCR assay were performed in a monolayer of MA-104 cells, which were grown in Dulbecco's Modified Eagle's medium (DMEM; Gibco BRL, Grand Island, NY, USA) supplemented with 10\% foetal calf serum (Gibco BRL, Carlsbad, CA, USA).

The inoculum was prepared as follows. A $10 \%(\mathrm{w} / \mathrm{v})$ faecal suspension in PBS solution was pretreated with $3 \times$ penicillin, streptomycin, and fungizone (Gibco Antibiotic-Antimycotic, Invitrogen Life Technologies, Carlsbad, CA, USA) for $24 \mathrm{~h}$. Then, the suspension was supplemented with $10 \mu \mathrm{g} / \mathrm{mL}$ trypsin and incubated at $37^{\circ} \mathrm{C}$ for $30 \mathrm{~min}$. Before inoculation, the confluent monolayer cells were washed 2-3 times with serum-free DMEM. Then, $1 \mathrm{~mL}$ of the treated suspension was inoculated onto the cell monolayer. The inoculum was allowed to adsorb at $37^{\circ} \mathrm{C}$ for $3 \mathrm{~h}$ in a temperature-controlled rocker platform. The inoculated MA-104 cells were maintained with serum-free DMEM supplemented with $0.5 \mu \mathrm{g} / \mathrm{mL}$ trypsin in a $\mathrm{CO}_{2}$ incubator (Thermo Electron Corporation, Marietta, OH, USA) and inspected daily for CPE.

qRT-PCR assay. All RVB-positive samples in the RT-PCR assay from these outbreaks were subjected to qRT-PCR assay to quantify the corresponding viral load. The qRT-PCR assay was developed in this study to quantify RVB RNA in biological samples.

The primers qRVB1174-Fw (5'-TGTTAGTATCCGCATTTGCTG-3') and qRVB1269-Rv (5'-GGGTTTTAT TGCTTATTTTTTCG-3') were designed to amplify a 95 bp fragment of a specific region of the porcine RVB VP6 gene. The probe was defined as qRVB1206-pb (5'-FAM-TCCGGCGTCAGCTCCCAAAGG-MGB-3') (Applied Biosystems, Foster City, CA, USA).

The qRT-PCR assay was performed in a 7500 Fast Real-Time PCR System (Applied Biosystems, Foster City, CA, USA) using the SuperScript III Platinum One-Step Quantitative RT-PCR System (Invitrogen Life Technologies, Carlsbad, CA, USA). The qRT-PCR master mix was prepared following the manufacturer's instructions with a final volume of $25 \mu \mathrm{L}$, including $50 \mathrm{nM}$ Rox Reference Dye, $400 \mathrm{nM}$ each forward and reverse primer, $200 \mathrm{nM}$ probe and $5 \mu \mathrm{L}$ of genomic template. All the samples were tested in triplicate. The cycling conditions included reverse transcription for $15 \mathrm{~min}$ at $50^{\circ} \mathrm{C}, \mathrm{Taq}$ activation for $2 \mathrm{~min}$ at $95^{\circ} \mathrm{C}$, followed by 40 cycles of $15 \mathrm{~s}$ at $95^{\circ} \mathrm{C}$, and $30 \mathrm{~s}$ at $60^{\circ} \mathrm{C}$. Sterile ultrapure water was used as a negative control in all reactions.

The number of genome copies present in the RVB-positive samples was estimated by comparing the sample cycle threshold $\left(C_{\mathrm{t}}\right)$ value to standard curves. To generate the standard curves, a $95 \mathrm{bp}$-RVB VP6 fragment was cloned into the pCR 4-TOPO vector (Invitrogen Life Technologies, Carlsbad, CA, USA), according to the manufacturer's instructions, and ligation products were transformed into TOP10 competent E. coli. After transformation, E. coli insert-containing cultures were extracted and purified by PureLink Quick Plasmid Miniprep Kit (Invitrogen Life Technologies, Carlsbad, CA, USA) and then quantified by Qubit Fluorometer (Invitrogen Life Technologies, Eugene, OR, USA). Tenfold serial dilutions of the RVB VP6 clone containing $1.34 \times 10^{7}$ to $1.34 \times 10^{0}$ copies $/ \mu \mathrm{L}$ were used to generate the standard curve and to estimate the LOD of the qRT-PCR assay. The final concentration in the samples was adjusted based on the volume of nucleic acids used and was expressed per gram of faeces/intestine tissue.

Sequencing and phylogenetic analysis. To identify the G genotypes of the porcine RVB strains, a VP7specific RT-PCR was carried out as described by Kuga et al. ${ }^{16}$. Sixteen RVB-positive faecal samples were selected for sequence analysis, and at least one RVB field strain was chosen from each pig herd. The RT-PCR products of approximately $800 \mathrm{bp}$ were purified using the PureLink Quick Gel Extraction and PCR Purification Combo Kit (Invitrogen Life Technologies, Carlsbad, CA, USA), quantified with a Qubit Fluorometer (Invitrogen Life Technologies, Eugene, OR, USA), and sequenced using an ABI 3500 Genetic Analyser with a BigDye Terminator v3.1 Cycle Sequencing kit (Applied Biosystems, Foster City, CA, USA).

Sequence quality analyses and consensus were conducted using Phred and CAP3 software (https://aspar gin.cenargen.embrapa.br/phph/). Sequence similarity searches were performed with sequences deposited in GenBank using the Nucleotide Basic Local Alignment Search Tool (BLASTn) (https://blast.ncbi.nlm.nih.gov/ Blast.cgi). A phylogenetic tree based on nucleotide sequences of the VP7 gene of porcine RVB strains described in this study and representative strains of the 26 previously identified RVB G genotypes ${ }^{12}$ was created using the neighbour-joining method based on the Kimura two-parameter model, which provided statistical support by bootstrapping with 1,000 replicates in MEGA 7 software ${ }^{37}$. BioEdit software, version 7.2.5, was used to construct the sequence identity matrix ${ }^{38}$. The nt cutoff value used to classify the RVB strains into G genotypes was $80 \%$, as suggested by Shepherd et al. ${ }^{12}$ (accession numbers of all RVB G genotype strains used to construct the phylogenetic tree and the identity matrix can be found as Supplementary Table S3 online).

Nucleotide sequences accession numbers. The nucleotide sequences described in this study were deposited in the GenBank database under the following accession numbers: MN540126 to MN540141.

Ethical approval. This study was submitted to the Ethics Committee on Animal Experiments of the Universidade Estadual de Londrina and approved under the identification number 11363.2015.16. All applicable international, national, and/or institutional guidelines for the care and use of animals were followed.

Received: 7 July 2020; Accepted: 19 October 2020

Published online: 15 December 2020 


\section{References}

1. Martella, V., Bányai, K., Matthijnssens, J., Buonavoglia, C. \& Ciarlet, M. Zoonotic aspects of rotaviruses. Vet. Microbiol. 140, 246-255 (2010).

2. Estes, M. K. \& Greenberg, H. B. In Rotaviruses. Fields virology 6th edn (eds Knipe, D. M., Howley, P. M. et al.) 1347-1401 (Wolters Kluwer Health/Lippincott Williams \& Wilkins, Philadelphia, 2013).

3. Matthijnssens, J. et al. VP6-sequence-based cutoff values as a criterion for rotavirus species demarcation. Arch. Virol. 157, 11771182 (2012).

4. Crawford, S. E. et al. Rotavirus infection. Nat. Rev. Dis. Primer 3, 17083 (2017).

5. Bányai, K. et al. Candidate new rotavirus species in Schreiber's bats, Serbia. Infect. Genet. Evol. 48, 19-26 (2017).

6. Molinari, B. L. D., Possatti, F., Lorenzetti, E., Alfieri, A. F. \& Alfieri, A. A. Unusual outbreak of post-weaning porcine diarrhea caused by single and mixed infections of rotavirus groups A, B, C, and H. Vet. Microbiol. 193, 125-132 (2016).

7. Marthaler, D. et al. Rapid detection and high occurrence of porcine rotavirus A, B, and C by RT-qPCR in diagnostic samples. J. Virol. Methods 209, 30-34 (2014).

8. Rotavirus Classification Working Group: RCWG-laboratory of viral metagenomics. https://rega.kuleuven.be/cev/viralmetag enomics/virus-classification/rcwg. Accessed 1 Oct 2020 (2020).

9. Matthijnssens, J. et al. Full genome-based classification of rotaviruses reveals a common origin between human Wa-Like and porcine rotavirus strains and human DS-1-like and bovine rotavirus strains. J. Virol. 82, 3204-3219 (2008).

10. Matthijnssens, J. et al. Uniformity of rotavirus strain nomenclature proposed by the Rotavirus Classification Working Group (RCWG). Arch. Virol. 156, 1397-1413 (2011).

11. Marthaler, D. et al. Detection of substantial porcine group B rotavirus genetic diversity in the United States, resulting in a modified classification proposal for G genotypes. Virology 433, 85-96 (2012).

12. Shepherd, F. K. et al. Whole genome classification and phylogenetic analyses of rotavirus B strains from the United States. Pathogens 7, 1-15 (2018).

13. Médici, K. C., Barry, A. F., Alfieri, A. F. \& Alfieri, A. A. Porcine rotavirus groups A, B, and C identified by polymerase chain reaction in a fecal sample collection with inconclusive results by polyacrylamide gel electrophoresis. J. Swine Heal. Prod. 19, 146-150 (2011).

14. Lahon, A., Ingle, V. C., Birade, H. S., Raut, C. G. \& Chitambar, S. D. Molecular characterization of group B rotavirus circulating in pigs from India: Identification of a strain bearing a novel VP7 genotype, G21. Vet. Microbiol. 174, 342-352 (2014).

15. Otto, P. H. et al. Detection of rotavirus species A, B and C in domestic mammalian animals with diarrhoea and genotyping of bovine species A rotavirus strains. Vet. Microbiol. 179, 168-176 (2015).

16. Kuga, K. et al. Genetic diversity and classification of the outer capsid glycoprotein VP7 of porcine group B rotaviruses. Arch. Virol. 154, 1785-1795 (2009).

17. Leme, R. A., Oliveira, T. E. S., Alfieri, A. F., Headley, S. A. \& Alfieri, A. A. Pathological, immunohistochemical and molecular findings associated with senecavirus A-induced lesions in neonatal piglets. J. Comp. Pathol. 155, 145-155 (2016).

18. Xia, L., Yang, Y., Wang, J., Jing, Y. \& Yang, Q. Impact of TGEV infection on the pig small intestine. Virol. J. 15, 102 (2018).

19. Oliveira, T. E. S. et al. Histopathological, immunohistochemical, and ultrastructural evidence of spontaneous Senecavirus A-induced lesions at the choroid plexus of newborn piglets. Sci. Rep. 7, 1-11 (2017).

20. Ramig, R. F. Pathogenesis of intestinal and systemic rotavirus infection. J. Virol. 78, 10213-10220 (2004).

21. Chang, K.-O., Saif, L. J. \& Kim, Y. Reoviruses (rotaviruses and reoviruses). in (eds. Zimmerman, J.J. et al.) Diseases of Swines, 10th edn 621-634 (Wiley/Blackwell, Ames, 2012).

22. Alekseev, K. et al. Genome characterization of a pathogenic porcine rotavirus B strain identified in Buryat Republic, Russia in 2015. Pathogens 7, 46 (2018).

23. Lorenzetti, E. et al. Diarrhea outbreaks in suckling piglets due to rotavirus group C single and mixed (rotavirus groups A and B) infections. Pesqui. Vet. Bras. 34, 391-397 (2014).

24. Possatti, F., Lorenzetti, E., Alfieri, A. F. \& Alfieri, A. A. Genetic heterogeneity of the VP6 gene and predominance of G6P[5] genotypes of Brazilian porcine rotavirus C field strains. Arch. Virol. 161, 1061-1067 (2016).

25. Flewett, T. H. Rotavirus in the home and hospital nursery. BMJ 287, 568-569 (1983).

26. Simpson, R., Aliyu, S., Iturriza-Gómara, M., Desselberger, U. \& Gray, J. Infantile viral gastroenteritis: On the way to closing the diagnostic gap. J. Med. Virol. 70, 258-262 (2003).

27. Ibrahim, O. S., Sunderland, D. \& Hart, C. A. Comparison of four methods for detection of rotavirus in faeces. Trop. Doct. 20, 30-32 (1990).

28. Sanekata, T. et al. Isolation of group B porcine rotavirus in cell culture. J. Clin. Microbiol. 34, 759-761 (1996).

29. Wakuda, M. et al. Porcine rotavirus closely related to novel group of human rotaviruses. Emerg. Infect. Dis. 17, 1491-1493 (2011).

30. Suzuki, T., Kuga, K., Miyazaki, A. \& Tsunemitsu, H. Genetic divergence and classification of non-structural protein 1 among porcine rotaviruses of species B. J. Gen. Virol. 92, 2922-2929 (2011).

31. Suzuki, T., Soma, J., Miyazaki, A. \& Tsunemitsu, H. Phylogenetic analysis of nonstructural protein 5 (NSP5) gene sequences in porcine rotavirus B strains. Infect. Genet. Evol. 12, 1661-1668 (2012).

32. Suzuki, T., Soma, J., Kuga, K., Miyazaki, A. \& Tsunemitsu, H. Sequence and phylogenetic analyses of nonstructural protein 2 genes of species B porcine rotaviruses detected in Japan during 2001-2009. Virus Res. 165, 46-51 (2012).

33. Marthaler, D. et al. VP6 genetic diversity, reassortment, intragenic recombination and classification of rotavirus B in American and Japanese pigs. Vet. Microbiol. 172, 359-366 (2014).

34. Molinari, B. L. D., Alfieri, A. F. \& Alfieri, A. A. Molecular characterization of a new G (VP7) genotype in group B porcine rotavirus. Intervirology 61, 42-48 (2018).

35. Marthaler, D. et al. Identification, phylogenetic analysis and classification of porcine group C rotavirus VP7 sequences from the United States and Canada. Virology 446, 189-198 (2013).

36. Alfieri, A. A., Parazzi, M. E., Takiuchi, E., Médici, K. C. \& Alfieri, A. F. Frequency of group A rotavirus in diarrhoeic calves in Brazilian cattle herds, 1998-2002. Trop. Anim. Health Prod. 38, 521-526 (2006).

37. Kumar, S., Stecher, G., Tamura, K. \& Dudley, J. MEGA7: Molecular evolutionary genetics analysis version 70 for bigger datasets. Mol. Biol. Evol. 33, 1870-1874 (2016).

38. Hall, T. A. BioEdit: A user-friendly biological sequence alignment editor and analysis program for Windows 95/98/NT. Nucl. Acids. Symp. 95-98. https://www.scienceopen.com/document?vid=8b59b929-3c37-49f6-936b-f8bf6dd92ace. Accessed 7 July 2020 (1999).

39. Gentsch, J. R. et al. Identification of group A rotavirus gene 4 types by polymerase chain reaction. J. Clin. Microbiol. 30, 1365-1373 (1992).

40. Gouvea, V. et al. Polymerase chain reaction amplification and typing of rotavirus nucleic acid from stool specimens. J. Clin. Microbiol. 28, 276-282 (1990).

41. Gouvea, V. et al. Detection of group B and C rotaviruses by polymerase chain reaction. J. Clin. Microbiol. 29, 519-523 (1991).

42. Alfieri, A. A. et al. Detection of field isolates of human and animal group C rotavirus by reverse transcription-polymerase chain reaction and digoxigenin-labeled oligonucleotide probes. J. Virol. Methods 83, 35-43 (1999).

43. Molinari, B. L. D., Alfieri, A. F. \& Alfieri, A. A. Genetic variability of VP6, VP7, VP4, and NSP4 genes of porcine rotavirus group $\mathrm{H}$ detected in Brazil. Virus Res. 197, 48-53 (2015). 
44. Wang, L., Byrum, B. \& Zhang, Y. Detection and genetic characterization of deltacoronavirus in pigs, Ohio, USA, 2014. Emerg. Infect. Dis. 20, 1227-1230 (2014).

45. Kim, S. Y., Song, D. S. \& Park, B. K. Differential detection of transmissible gastroenteritis virus and porcine epidemic diarrhea virus by duplex RT-PCR. J. Vet. Diagn. Invest. 13, 516-520 (2001).

46. Feronato, C. et al. Development and evaluation of a nested-PCR assay for Senecavirus A diagnosis. Trop. Anim. Health Prod. 50, 337-344 (2018).

47. Krumbholz, A. et al. Detection of porcine teschoviruses and enteroviruses by LightCycler real-time PCR. J. Virol. Methods 113, 51-63 (2003).

\section{Acknowledgements}

This study was supported by the following Brazilian institutions: The National Council for Scientific and Technological Development (CNPq), the Coordination for the Improvement of Higher Education Personnel (CAPES), and the Araucaria Foundation (FAP/PR). Alfieri, A.A., Alfieri, A.F., Headley, S.A, Oliveira, T.E.S, and Miyabe, F.M. are recipients of CNPq fellowships. A.M. Dall Agnol is recipient of INCT-Leite/CAPES fellowship (grant number 88887.495081/2020-00).

\section{Author contributions}

F.M.M., R.A.L., and A.A.A. contributed to the conception and design of the study, the analysis and interpretation of all virological and molecular data. F.M.M. wrote the manuscript. R.A.L., A.M.D.A., A.F.A. and A.A.A. contributed to the acquisition of all samples and review of the manuscript. T.E.S.O. and S.A.H. contributed to the preparation, evaluation and analyses of the histopathological data. T.F., and A.G.O. contributed to the preparation, evaluation, and analyses of EM data. All authors have read, critically analysed and approved the final draft of this manuscript.

\section{Competing interests}

The authors declare no competing interests.

\section{Additional information}

Supplementary Information The online version contains supplementary material available at https://doi. org/10.1038/s41598-020-78797-y.

Correspondence and requests for materials should be addressed to A.A.A.

Reprints and permissions information is available at www.nature.com/reprints.

Publisher's note Springer Nature remains neutral with regard to jurisdictional claims in published maps and institutional affiliations.

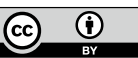

Open Access This article is licensed under a Creative Commons Attribution 4.0 International License, which permits use, sharing, adaptation, distribution and reproduction in any medium or format, as long as you give appropriate credit to the original author(s) and the source, provide a link to the Creative Commons licence, and indicate if changes were made. The images or other third party material in this article are included in the article's Creative Commons licence, unless indicated otherwise in a credit line to the material. If material is not included in the article's Creative Commons licence and your intended use is not permitted by statutory regulation or exceeds the permitted use, you will need to obtain permission directly from the copyright holder. To view a copy of this licence, visit http://creativecommons.org/licenses/by/4.0/.

(C) The Author(s) 2020 